\title{
La microscopie du vivant : de nouveaux outils
}

\section{Les nouvelles de ce numéro ont été préparées par : Pascale Briand Jean-Claude Dreyfus Axel Kahn Marc Peschanski}

1. Golovchenki JA. The tunneling microscope: a new look at the atomic world Science 1986 ; 232 : 48-53.

2. Sonnenfeld R, Hansma PK. Atomic-resolution microscopy in water. Science 1986 ; 232 : $211-3$

3. Pethica J. New ways to see atoms. Nature 1987 ; 325 : 388-9.

4. Amrein M, Stasiak A, Gross H, Travaglini G. Scanning tunneling microscopy of recADNA complexes coated with conducting film. Science $1988 ; 240: 514-6$

5. Gould S, Marti O, Drake B, et al. Molecular resolution images of amino-acid crystals with the atomic force microscope. Nature $1988 ; 332 ; 332-4$

6. Cudney RS, Pierce Rm, Feinberg J. The transient detection microscope. Nature 1988 ; 332 : 424-6.

$\mathrm{m} / \mathrm{s} n^{\circ} 7$ vol. 4 , septembre 88
Sous le titre «visualiser des molécules en action », une nouvelle parue dans médecine/science ( $\mathrm{m} / \mathrm{s} n^{\circ} 7$, vol. 2, p. 401) décrivait, il y a moins de deux ans, le «microscope à effet tunnel» (scanning tunneling microscope: STM) [1] qui vit quelques semaines plus tard ses inventeurs Binnig et Rohrer, recevoir le Prix Nobel de Physique. Les premiers résultats concernant son utilisation pour l'étude d'échantillons placés en milieux aqueux [2] permettaient déjà d'être optimiste quant à ses applications en biologie. Un an plus tard, le STM était supplanté par l'AFM (atomic force microscope) dont le principe est similaire à celui du STM, à ceci près que le tracé des contours est dû à la force constante qui existe entre la pointe du détecteur et la surface à «visualiser» et non plus à celui du courant, lui aussi constant, dû à l'effet tunnel [3]. Si la résolution, perpendiculairement à la surface, est du même ordre de grandeur qu'avec le STM (inférieure à l'Angström) la résolution latérale est améliorée d'un facteur 10 atteignant deux à trois Angström. L'un des avantages essentiels de l'AFM est que contrairement au STM, la surface à étudier peut ne pas être conductrice, ouvrant le champ à l'étude de nombreux composés biologiques dont la conductivité n'est pas connue (ce qui, le plus souvent, est le cas à l'échelle d'une molécule). Très récemment des complexes protéine recA-ADN recouverts d'un film conducteur furent visualisés grâce au STM [4]. La résolution obtenue est à l'évidence supérieure à celle du microscope électronique et les résultats sont si reproductibles qu'il n'est point besoin d'effectuer de moyennes sur plusieurs observations. Dans le cas par exemple des complexes protéine recA-ADN, le STM permet de déterminer le nombre de monomères recA par tour d'hélice sans effectuer de moyenne sur plusieurs observations. La structure tridimentionnelle peut de ce fait être plus facilement reconstruite. Grâce cette fois à l'AFM, des acides aminés furent aussi visualisés [5]. Cette première analyse fut effectuée sur un cristal de DL-leucine, permettant ainsi la comparaison des résultats à ceux obtenus par la diffraction aux rayons $\mathrm{X}$, mais cette technique est aussi applicable à des molécules biologiques non cristallisables.

Basé sur un tout autre principe puisqu'il s'agir d'un microscope à laser, le transient detection microscope (microscope détecteur des effets transitoires) permet de ne visualiser que les objets en mouvement. Le principe en est simple : un faisceau laser éclaire l'objet qui est séparé de l'objectif ou de la caméra par un cristal de titanate de barium. Un second faisceau laser, dit de référence, cohérent avec le premier, est envoyé directement sur le cristal. Les interférences optiques résultantes vont créer un hologramme au sein du cristal. Une fraction du faisceau de référence est diffractée dans la direction du faisceau transmis par l'objet et une fraction du faisceau ayant traversé l'objet suit la direction du faisceau de référence. L'on peut choisir des paramètres (angles et énergie des faisceaux, type et orientation du crystal...) tels que la presque totalité de l'énergie du faisceau qui a traversé l'objet soit captée par le faisceau de référence. L'image obtenue est alors pratiquement noire. Il faut un certain temps, dépendant des caractéristiques du cristal et de l'intensité des faisceaux, pour que s'établissent ces interférences destructrices. Si un mouvement se produit dans l'objet, à une vitesse telle que l'état d'interférence qui aboutit à l'élimination de l'image n'ait pas eu le temps de se mettre en place, une image apparaît. Des protozoaires dont la taille varie de 5 à $30 \mu \mathrm{m}$ peuvent ainsi, dans le système décrit par les auteurs, être visualisés lorsque leur vitesse de déplacement est supérieure à $25 \mu \mathrm{m} / \mathrm{s}$. En changeant simplement l'axe du cristal ce microscope peut aussi être utilisé pour intensifier l'image d'objets immobiles.

P. B. 